\title{
A Determination of the Centre-of-Mass Energy at LEP2 using Radiative 2-fermion Events
}

\author{
DELPHI Collaboration
}

\begin{abstract}
Using $\mathrm{e}^{+} \mathrm{e}^{-} \rightarrow \mu^{+} \mu^{-}(\gamma)$ and $\mathrm{e}^{+} \mathrm{e}^{-} \rightarrow \mathrm{q} \overline{\mathrm{q}}(\gamma)$ events radiative to the $\mathrm{Z}$ pole, DELPHI has determined the centre-of-mass energy, $\sqrt{s}$, using energy and momentum constraint methods. The results are expressed as deviations from the nominal LEP centre-of-mass energy, measured using other techniques. The results are found to be compatible with the LEP Energy Working Group estimates for a combination of the 1997 to 2000 data sets.
\end{abstract}




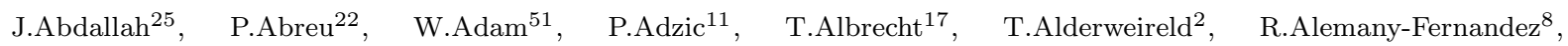
T.Allmendinger ${ }^{17}$, P.P.Allport ${ }^{23}$, U.Amaldi ${ }^{29}$, N.Amapane ${ }^{45}$, S.Amato ${ }^{48}$, E.Anashkin ${ }^{36}$, A.Andreazza ${ }^{28}$, S.Andringa ${ }^{22}$, N.Anjos ${ }^{22}$, P.Antilogus ${ }^{25}$, W-D.Apel ${ }^{17}$, Y.Arnoud ${ }^{14}$, S.Ask ${ }^{26}$, B.Asman ${ }^{44}$, J.E.Augustin ${ }^{25}$, A.Augustinus ${ }^{8}$, P.Baillon ${ }^{8}$, A.Ballestrero ${ }^{46}$, P.Bambade ${ }^{20}$, R.Barbier ${ }^{27}$, D.Bardin ${ }^{16}$, G.J.Barker ${ }^{17}$, A.Baroncelli ${ }^{39}$, M.Battaglia ${ }^{8}$, M.Baubillier ${ }^{25}$, K-H.Becks ${ }^{53}$, M.Begalli ${ }^{6}$, A.Behrmann ${ }^{53}$, E.Ben-Haim ${ }^{20}$, N.Benekos ${ }^{32}$, A.Benvenuti ${ }^{5}$, C.Berat ${ }^{14}$, M.Berggren ${ }^{25}$, L.Berntzon $^{44}$, D.Bertrand ${ }^{2}$, M.Besancon ${ }^{40}$, N.Besson ${ }^{40}$, D.Bloch ${ }^{9}$, M.Blom ${ }^{31}$, M.Bluj ${ }^{52}$, M.Bonesini ${ }^{29}$, M.Boonekamp ${ }^{40}$, P.S.L.Booth ${ }^{\dagger 23}$, G.Borisov ${ }^{21}$, O.Botner ${ }^{49}$, B.Bouquet ${ }^{20}$, T.J.V.Bowcock ${ }^{23}$, I.Boyko ${ }^{16}$, M.Bracko ${ }^{43}$, R.Brenner ${ }^{49}$, E.Brodet ${ }^{35}$, P.Bruckman ${ }^{18}$, J.M.Brunet ${ }^{7}$, B.Buschbeck ${ }^{51}$, P.Buschmann ${ }^{53}$, M.Calvi ${ }^{29}$, T.Camporesi ${ }^{8}$, V.Canale ${ }^{38}$, F.Carena ${ }^{8}$, N.Castro ${ }^{22}$, F.Cavallo ${ }^{5}$, M.Chapkin ${ }^{42}$, Ph.Charpentier ${ }^{8}$, P.Checchia ${ }^{36}$, R.Chierici ${ }^{8}$, P.Chliapnikov ${ }^{42}$, J.Chudoba $^{8}$, S.U.Chung ${ }^{8}$, K.Cieslik ${ }^{18}$, P.Collins ${ }^{8}$, R.Contri ${ }^{13}$, G.Cosme ${ }^{20}$, F.Cossutti ${ }^{47}$, M.J.Costa ${ }^{50}$, D.Crennell ${ }^{37}$, J.Cuevas $^{34}$, J.D'Hondt ${ }^{2}$, J.Dalmau ${ }^{44}$, T.da Silva ${ }^{48}$, W.Da Silva ${ }^{25}$, G.Della Ricca ${ }^{47}$, A.De Angelis ${ }^{47}$, W.De Boer ${ }^{17}$, C.De Clercq ${ }^{2}$, B.De Lotto ${ }^{47}$, N.De Maria ${ }^{45}$, A.De Min ${ }^{36}$, L.de Paula ${ }^{48}$, L.Di Ciaccio ${ }^{38}$, A.Di Simone ${ }^{39}$, K.Doroba ${ }^{52}$, J.Drees $^{53,8}$, G.Eigen ${ }^{4}$, T.Ekelof ${ }^{49}$, M.Ellert ${ }^{49}$, M.Elsing ${ }^{8}$, M.C.Espirito Santo ${ }^{22}$, G.Fanourakis ${ }^{11}$, D.Fassouliotis ${ }^{11,3}$, M.Feindt ${ }^{17}$, J.Fernandez ${ }^{41}$, A.Ferrer ${ }^{50}$, F.Ferro ${ }^{13}$, U.Flagmeyer ${ }^{53}$, H.Foeth ${ }^{8}$, E.Fokitis ${ }^{32}$, F.Fulda-Quenzer ${ }^{20}$, J.Fuster ${ }^{50}$, M.Gandelman ${ }^{48}$, C.Garcia ${ }^{50}$, Ph.Gavillet ${ }^{8}$, E.Gazis ${ }^{32}$, R.Gokieli ${ }^{8,52}$, B.Golob ${ }^{43}$, G.Gomez-Ceballos ${ }^{41}$, P.Goncalves ${ }^{22}$, E.Graziani ${ }^{39}$, G.Grosdidier ${ }^{20}$, K.Grzelak ${ }^{52}$, J.Guy ${ }^{37}$, C.Haag ${ }^{17}$, A.Hallgren ${ }^{49}$, K.Hamacher ${ }^{53}$, K.Hamilton ${ }^{35}$, S.Haug ${ }^{33}$, F.Hauler ${ }^{17}$, V.Hedberg ${ }^{26}$, M.Hennecke ${ }^{17}$, H.Herr ${ }^{\dagger 8}$, J.Hoffman ${ }^{52}$, S-O.Holmgren ${ }^{44}$, P.J.Holt ${ }^{8}$, M.A.Houlden ${ }^{23}$, K.Hultqvist ${ }^{44}$, J.N.Jackson ${ }^{23}$, G.Jarlskog ${ }^{26}$, P.Jarry ${ }^{40}$, D.Jeans ${ }^{35}$, E.K.Johansson ${ }^{44}$, P.D.Johansson ${ }^{44}$, P.Jonsson ${ }^{27}$, C.Joram ${ }^{8}$, L.Jungermann ${ }^{17}$, F.Kapusta ${ }^{25}$, S.Katsanevas ${ }^{27}$, E.Katsoufis ${ }^{32}$, G.Kernel ${ }^{43}$, B.P.Kersevan ${ }^{8,43}$, U.Kerzel ${ }^{17}$, B.T.King ${ }^{23}$, N.J.Kjaer ${ }^{8}$, P.Kluit ${ }^{31}$, P.Kokkinias ${ }^{11}$, C.Kourkoumelis ${ }^{3}$, O.Kouznetsov ${ }^{16}$, Z.Krumstein ${ }^{16}$, M.Kucharczyk ${ }^{18}$, J.Lamsa $^{1}$, G.Leder ${ }^{51}$, F.Ledroit ${ }^{14}$, L.Leinonen ${ }^{44}$, R.Leitner ${ }^{30}$, J.Lemonne ${ }^{2}$, V.Lepeltier ${ }^{20}$, T.Lesiak ${ }^{18}$, W.Liebig ${ }^{53}$, D.Liko ${ }^{51}$, A.Lipniacka ${ }^{44}$, J.H.Lopes ${ }^{48}$, J.M.Lopez ${ }^{34}$, D.Loukas ${ }^{11}$, P.Lutz ${ }^{40}$, L.Lyons ${ }^{35}$, J.MacNaughton ${ }^{51}$, A.Malek ${ }^{53}$, S.Maltezos $^{32}$, F.Mandl ${ }^{51}$, J.Marco ${ }^{41}$, R.Marco ${ }^{41}$, B.Marechal ${ }^{48}$, M.Margoni ${ }^{36}$, J-C.Marin ${ }^{8}$, C.Mariotti ${ }^{8}$, A.Markou ${ }^{11}$, C.Martinez-Rivero $^{41}$, J.Masik ${ }^{12}$, N.Mastroyiannopoulos ${ }^{11}$, F.Matorras ${ }^{41}$, $\quad$ C.Matteuzzi $^{29}$, F.Mazzucato ${ }^{36}$, M.Mazzucato $^{36}$, R.Mc Nulty ${ }^{23}$, C.Meroni ${ }^{28}$, E.Migliore ${ }^{45}$, W.Mitaroff ${ }^{51}$, U.Mjoernmark ${ }^{26}$, T.Moa ${ }^{44}$, M.Moch $^{17}$, K.Moenig ${ }^{8,10}$, R.Monge ${ }^{13}$, J.Montenegro ${ }^{31}$, D.Moraes ${ }^{48}$, S.Moreno ${ }^{22}$, P.Morettini ${ }^{13}$, U.Mueller ${ }^{53}$, K.Muenich ${ }^{53}$, M.Mulders ${ }^{31}$, L.Mundim ${ }^{6}$, W.Murray ${ }^{37}$, B.Muryn ${ }^{19}$, G.Myatt ${ }^{35}$, T.Myklebust ${ }^{33}$, M.Nassiakou ${ }^{11}$, F.Navarria ${ }^{5}$, K.Nawrocki ${ }^{52}$, R.Nicolaidou ${ }^{40}$, M.Nikolenko ${ }^{16,9}$, A.Oblakowska-Mucha ${ }^{19}$, V.Obraztsov ${ }^{42}$, A.Olshevski ${ }^{16}$, A.Onofre ${ }^{22}$, R.Orava ${ }^{15}$, K.Osterberg ${ }^{15}$, A.Ouraou ${ }^{40}$, A.Oyanguren ${ }^{50}$, M.Paganoni ${ }^{29}$, S.Paiano ${ }^{5}$, J.P.Palacios ${ }^{23}$, H.Palka ${ }^{18}$, Th.D.Papadopoulou ${ }^{32}$, L.Pape ${ }^{8}$, C.Parkes ${ }^{24}$, F.Parodi ${ }^{13}$, U.Parzefall ${ }^{8}$, A.Passeri ${ }^{39}$, O.Passon ${ }^{53}$, L.Peralta ${ }^{22}$, V.Perepelitsa ${ }^{50}$, A.Perrotta ${ }^{5}$, A.Petrolini ${ }^{13}$, J.Piedra ${ }^{41}$, L.Pieri ${ }^{39}$, F.Pierre ${ }^{40}$, M.Pimenta ${ }^{22}$, E.Piotto ${ }^{8}$, T.Podobnik ${ }^{43}$, V.Poireau ${ }^{8}$, M.E.Pol ${ }^{6}$, G.Polok ${ }^{18}$, V.Pozdniakov ${ }^{16}$, N.Pukhaeva ${ }^{2,16}$, A.Pullia ${ }^{29}$, J.Rames ${ }^{12}$, A.Read ${ }^{33}$, P.Rebecchi ${ }^{8}$, J.Rehn ${ }^{17}$, D.Reid ${ }^{31}$, R.Reinhardt ${ }^{53}$, P.Renton ${ }^{35}$, F.Richard ${ }^{20}$, J.Ridky ${ }^{12}$, M.Rivero ${ }^{41}$, D.Rodriguez ${ }^{41}$, A.Romero ${ }^{45}$, P.Ronchese $^{36}$, P.Roudeau ${ }^{20}$, T.Rovelli ${ }^{5}$, V.Ruhlmann-Kleider ${ }^{40}$, D.Ryabtchikov ${ }^{42}$, A.Sadovsky ${ }^{16}$, L.Salmi ${ }^{15}$, J.Salt ${ }^{50}$, C.Sander ${ }^{17}$, A.Savoy-Navarro ${ }^{25}$, U.Schwickerath ${ }^{8}$, A.Segar ${ }^{\dagger 5}$, R.Sekulin ${ }^{37}$, M.Siebel ${ }^{53}$, A.Sisakian ${ }^{16}$, G.Smadja ${ }^{27}$, O.Smirnova ${ }^{26}$, A.Sokolov ${ }^{42}$, A.Sopczak ${ }^{21}$, R.Sosnowski ${ }^{52}$, T.Spassov ${ }^{8}$, M.Stanitzki ${ }^{17}$, A.Stocchi ${ }^{20}$, J.Strauss ${ }^{51}$, B.Stugu ${ }^{4}$, M.Szczekowski ${ }^{52}$, M.Szeptycka ${ }^{52}$, T.Szumlak ${ }^{19}$, T.Tabarelli ${ }^{29}$, A.C.Taffard ${ }^{23}$, F.Tegenfeldt ${ }^{49}$, J.Timmermans ${ }^{31}$, L.Tkatchev ${ }^{16}$, M.Tobin ${ }^{23}$, S.Todorovova ${ }^{12}$, B.Tome ${ }^{22}$, A.Tonazzo ${ }^{29}$, P.Tortosa ${ }^{50}$, P.Travnicek ${ }^{12}$, D.Treille ${ }^{8}$, G.Tristram ${ }^{7}$, M.Trochimczuk ${ }^{52}$, C.Troncon ${ }^{28}$, M-L.Turluer ${ }^{40}$, I.A.Tyapkin ${ }^{16}$, P.Tyapkin ${ }^{16}$, S.Tzamarias ${ }^{11}$, V.Uvarov ${ }^{42}$, G.Valenti ${ }^{5}$, P.Van Dam ${ }^{31}$, J.Van Eldik ${ }^{8}$, N.van Remortel ${ }^{15}$, I.Van Vulpen ${ }^{8}$, G.Vegni ${ }^{28}$, F.Veloso ${ }^{22}$, W.Venus ${ }^{37}$, P.Verdier ${ }^{27}$, V.Verzi $^{38}$, D.Vilanova ${ }^{40}$, L.Vitale ${ }^{47}$, V.Vrba ${ }^{12}$, H.Wahlen ${ }^{53}$, A.J.Washbrook ${ }^{23}$, C.Weiser ${ }^{17}$, D.Wicke ${ }^{8}$, J.Wickens ${ }^{2}$, G.Wilkinson ${ }^{35}$, M.Winter ${ }^{9}$, M.Witek ${ }^{18}$, O.Yushchenko ${ }^{42}$, A.Zalewska ${ }^{18}$, P.Zalewski ${ }^{52}$, D.Zavrtanik ${ }^{43}$, V.Zhuravlov ${ }^{16}$, N.I.Zimin ${ }^{16}$, A.Zintchenko ${ }^{16}$, M.Zupan ${ }^{11}$ 


\footnotetext{
${ }^{1}$ Department of Physics and Astronomy, Iowa State University, Ames IA 50011-3160, USA

${ }^{2}$ Physics Department, Universiteit Antwerpen, Universiteitsplein 1, B-2610 Antwerpen, Belgium and IIHE, ULB-VUB, Pleinlaan 2, B-1050 Brussels, Belgium

and Faculté des Sciences, Univ. de l'Etat Mons, Av. Maistriau 19, B-7000 Mons, Belgium

${ }^{3}$ Physics Laboratory, University of Athens, Solonos Str. 104, GR-10680 Athens, Greece

${ }^{4}$ Department of Physics, University of Bergen, Allégaten 55, NO-5007 Bergen, Norway

${ }^{5}$ Dipartimento di Fisica, Università di Bologna and INFN, Via Irnerio 46, IT-40126 Bologna, Italy

${ }^{6}$ Centro Brasileiro de Pesquisas Físicas, rua Xavier Sigaud 150, BR-22290 Rio de Janeiro, Brazil and Depto. de Física, Pont. Univ. Católica, C.P. 38071 BR-22453 Rio de Janeiro, Brazil and Inst. de Física, Univ. Estadual do Rio de Janeiro, rua São Francisco Xavier 524, Rio de Janeiro, Brazil ${ }^{7}$ Collège de France, Lab. de Physique Corpusculaire, IN2P3-CNRS, FR-75231 Paris Cedex 05, France ${ }^{8}$ CERN, CH-1211 Geneva 23, Switzerland

${ }^{9}$ Institut de Recherches Subatomiques, IN2P3 - CNRS/ULP - BP20, FR-67037 Strasbourg Cedex, France

${ }^{10}$ Now at DESY-Zeuthen, Platanenallee 6, D-15735 Zeuthen, Germany

${ }^{11}$ Institute of Nuclear Physics, N.C.S.R. Demokritos, P.O. Box 60228, GR-15310 Athens, Greece

${ }^{12} \mathrm{FZU}$, Inst. of Phys. of the C.A.S. High Energy Physics Division, Na Slovance 2, CZ-180 40, Praha 8, Czech Republic

${ }^{13}$ Dipartimento di Fisica, Università di Genova and INFN, Via Dodecaneso 33, IT-16146 Genova, Italy

${ }^{14}$ Institut des Sciences Nucléaires, IN2P3-CNRS, Université de Grenoble 1, FR-38026 Grenoble Cedex, France

${ }^{15}$ Helsinki Institute of Physics and Department of Physical Sciences, P.O. Box 64, FIN-00014 University of Helsinki, Finland

${ }^{16}$ Joint Institute for Nuclear Research, Dubna, Head Post Office, P.O. Box 79, RU-101 000 Moscow, Russian Federation

${ }^{17}$ Institut für Experimentelle Kernphysik, Universität Karlsruhe, Postfach 6980, DE-76128 Karlsruhe, Germany

${ }^{18}$ Institute of Nuclear Physics PAN,Ul. Radzikowskiego 152, PL-31142 Krakow, Poland

${ }^{19}$ Faculty of Physics and Nuclear Techniques, University of Mining and Metallurgy, PL-30055 Krakow, Poland

${ }^{20}$ Université de Paris-Sud, Lab. de l'Accélérateur Linéaire, IN2P3-CNRS, Bât. 200, FR-91405 Orsay Cedex, France

${ }^{21}$ School of Physics and Chemistry, University of Lancaster, Lancaster LA1 4YB, UK

${ }^{22}$ LIP, IST, FCUL - Av. Elias Garcia, 14-1 ${ }^{\circ}$, PT-1000 Lisboa Codex, Portugal

${ }^{23}$ Department of Physics, University of Liverpool, P.O. Box 147, Liverpool L69 3BX, UK

${ }^{24}$ Dept. of Physics and Astronomy, Kelvin Building, University of Glasgow, Glasgow G12 8QQ

${ }^{25}$ LPNHE, IN2P3-CNRS, Univ. Paris VI et VII, Tour 33 (RdC), 4 place Jussieu, FR-75252 Paris Cedex 05, France

${ }^{26}$ Department of Physics, University of Lund, Sölvegatan 14, SE-223 63 Lund, Sweden

${ }^{27}$ Université Claude Bernard de Lyon, IPNL, IN2P3-CNRS, FR-69622 Villeurbanne Cedex, France

${ }^{28}$ Dipartimento di Fisica, Università di Milano and INFN-MILANO, Via Celoria 16, IT-20133 Milan, Italy

${ }^{29}$ Dipartimento di Fisica, Univ. di Milano-Bicocca and INFN-MILANO, Piazza della Scienza 2, IT-20126 Milan, Italy

${ }^{30}$ IPNP of MFF, Charles Univ., Areal MFF, V Holesovickach 2, CZ-180 00, Praha 8, Czech Republic

${ }^{31}$ NIKHEF, Postbus 41882, NL-1009 DB Amsterdam, The Netherlands

${ }^{32}$ National Technical University, Physics Department, Zografou Campus, GR-15773 Athens, Greece

${ }^{33}$ Physics Department, University of Oslo, Blindern, NO-0316 Oslo, Norway

${ }^{34}$ Dpto. Fisica, Univ. Oviedo, Avda. Calvo Sotelo s/n, ES-33007 Oviedo, Spain

${ }^{35}$ Department of Physics, University of Oxford, Keble Road, Oxford OX1 3RH, UK

${ }^{36}$ Dipartimento di Fisica, Università di Padova and INFN, Via Marzolo 8, IT-35131 Padua, Italy

${ }^{37}$ Rutherford Appleton Laboratory, Chilton, Didcot OX11 OQX, UK

${ }^{38}$ Dipartimento di Fisica, Università di Roma II and INFN, Tor Vergata, IT-00173 Rome, Italy

${ }^{39}$ Dipartimento di Fisica, Università di Roma III and INFN, Via della Vasca Navale 84, IT-00146 Rome, Italy

${ }^{40}$ DAPNIA/Service de Physique des Particules, CEA-Saclay, FR-91191 Gif-sur-Yvette Cedex, France

${ }^{41}$ Instituto de Fisica de Cantabria (CSIC-UC), Avda. los Castros s/n, ES-39006 Santander, Spain

${ }^{42}$ Inst. for High Energy Physics, Serpukov P.O. Box 35, Protvino, (Moscow Region), Russian Federation

${ }^{43}$ J. Stefan Institute, Jamova 39, SI-1000 Ljubljana, Slovenia and Laboratory for Astroparticle Physics,

Nova Gorica Polytechnic, Kostanjeviska 16a, SI-5000 Nova Gorica, Slovenia,

and Department of Physics, University of Ljubljana, SI-1000 Ljubljana, Slovenia

${ }^{44}$ Fysikum, Stockholm University, Box 6730, SE-113 85 Stockholm, Sweden

${ }^{45}$ Dipartimento di Fisica Sperimentale, Università di Torino and INFN, Via P. Giuria 1, IT-10125 Turin, Italy

${ }^{46}$ INFN,Sezione di Torino and Dipartimento di Fisica Teorica, Università di Torino, Via Giuria 1, IT-10125 Turin, Italy

${ }^{47}$ Dipartimento di Fisica, Università di Trieste and INFN, Via A. Valerio 2, IT-34127 Trieste, Italy and Istituto di Fisica, Università di Udine, IT-33100 Udine, Italy

${ }^{48}$ Univ. Federal do Rio de Janeiro, C.P. 68528 Cidade Univ., Ilha do Fundão BR-21945-970 Rio de Janeiro, Brazil

${ }^{49}$ Department of Radiation Sciences, University of Uppsala, P.O. Box 535, SE-751 21 Uppsala, Sweden

${ }^{50}$ IFIC, Valencia-CSIC, and D.F.A.M.N., U. de Valencia, Avda. Dr. Moliner 50, ES-46100 Burjassot (Valencia), Spain

${ }^{51}$ Institut für Hochenergiephysik, Österr. Akad. d. Wissensch., Nikolsdorfergasse 18, AT-1050 Vienna, Austria

${ }^{52}$ Inst. Nuclear Studies and University of Warsaw, Ul. Hoza 69, PL-00681 Warsaw, Poland

${ }^{53}$ Fachbereich Physik, University of Wuppertal, Postfach 100 127, DE-42097 Wuppertal, Germany

$\dagger$ deceased
} 


\section{Introduction}

One of the main goals of LEP2 is the precise measurement of the mass of the W-boson, $\mathrm{M}_{\mathrm{W}}$. The aim, with the integrated luminosity collected, is a total experimental uncertainty of about $40 \mathrm{MeV} / \mathrm{c}^{2}$. One important contribution to the systematic uncertainty on $\mathrm{M}_{\mathrm{W}}$ comes from the accuracy with which the centre-of-mass energy, $\mathrm{E}_{\mathrm{CM}}$, can be measured. To match other statistical and systematic errors, the error on $\mathrm{E}_{\mathrm{CM}}$ at LEP2 should be below $30 \mathrm{MeV}$, equivalent to an error on the beam energy of less than $15 \mathrm{MeV}$. At LEP1, very accurate determinations of $\mathrm{E}_{\mathrm{CM}}$ were made which relied on precise beam energy $\left(\mathrm{E}_{\text {beam }}\right)$ measurements provided by the resonant depolarisation technique [1]. At LEP2 energies resonant depolarisation is no longer possible. Therefore a method using a magnetic extrapolation is employed, in which resonant depolarisation is used at beam energies of 44-61 GeV to calibrate NMR probes in selected dipole magnets. These probes are then used to estimate the beam energy at LEP2 physics energies by extrapolating from the lower calibration energies [2].

The uncertainty on the extrapolation procedure dominates the energy determination. Several methods have been developed by the LEP Energy Working Group to constrain this uncertainty [2]. These include the measurement of the beam deflection in a spectrometer, an analysis based on the evolution of the synchrotron tune with the RF voltage, and a comparison of the bending field as measured by the NMRs with that sensed in the ring as a whole by the flux loop. The assigned error on $\mathrm{E}_{\mathrm{CM}}$ from combining the results of these analyses is 20-25 MeV for the majority of LEP2 operation, with a larger error of about $40 \mathrm{MeV}$ in 2000, where in addition to the magnetic extrapolation itself, other uncertainties are significant [2].

The collision energy can also be measured in both hadronic and leptonic channels using the "radiative return" events. In these events the effective $\mathrm{e}^{+} \mathrm{e}^{-}$centre-of-mass energy is reduced to that of an on-shell Z-boson by initial-state radiation (ISR). The ALEPH collaboration performed a measurement based upon the latter method using just the $183 \mathrm{GeV}$ data [3]. Subsequently, measurements at this and higher energies have been carried out by the L3 [4] and OPAL [5] collaborations. This note describes a method that uses $\mathrm{e}^{+} \mathrm{e}^{-} \rightarrow \mu^{+} \mu^{-}(\gamma)$ and $\mathrm{e}^{+} \mathrm{e}^{-} \rightarrow \mathrm{q} \overline{\mathrm{q}}(\gamma)$ radiative return events to measure the difference between the centre-of-mass energy determined from radiative events and the value from the LEP Energy Working Group $\Delta \mathrm{E}_{\mathrm{CM}}$. This method, particularly if results from the four LEP experiments can be combined, provides a cross-check against large systematic effects in the LEP Energy Working Group determination. The dimuon channel is the easiest in which to study the radiative return peak due to its simple two-prong topology. However, the statistical power is much smaller than that available from hadronic radiative returns.

The analysis used the data of the four last years of LEP data taking with the collision energy ranging from 183 to $207 \mathrm{GeV}$. The total data sample was organized in 8 energy points: 183, 189, 192, 196, 200, 202, 205, $207 \mathrm{GeV}$. The measurement was performed at each energy point separately and at the end a combined result was produced. Details of the components of the DELPHI detector and their performances can be found in $[6,7]$. Data from the period after 1st September 2000, when part of the TPC, the main tracking device of DELPHI, had high voltage problems, were not included.

Simulation of signal and all relevant backgrounds was available for each energy. The $\mathrm{e}^{+} \mathrm{e}^{-} \rightarrow \mu^{+} \mu^{-}(\gamma)$ and $\mathrm{e}^{+} \mathrm{e}^{-} \rightarrow \mathrm{q} \overline{\mathrm{q}}(\gamma)$ events were simulated with the KK 4.14 generator [8]. Most of the four-fermion final states were produced with the program described in [9], based on the WPHACT 2.0 generator [10]. However, as discussed in [9], for the final states $\mathrm{e}^{+} \mathrm{e}^{-} \mathrm{f} \overline{\mathrm{f}}$, collisions with either the $\mathrm{e}^{+}$or $\mathrm{e}^{-}$emitted at less than $2^{\circ}$ to the 
beam, and with the invariant mass of the ff system less than $40 \mathrm{GeV} / c^{2}$ were generated with PYTHIA 6.143 [11]. Hadronisation was performed with PYTHIA 6.156. In addition, ARIADNE 4.08 [12] was used for fragmentation studies. The generated events were passed through the DELPHI detector simulation program DELSIM [7], and then through the same reconstruction and analysis programs as the data.

Section 2 describes the analysis of the $\mathrm{e}^{+} \mathrm{e}^{-} \rightarrow \mu^{+} \mu^{-}(\gamma)$ channel, and Section 3 that of the $\mathrm{e}^{+} \mathrm{e}^{-} \rightarrow \mathrm{q} \overline{\mathrm{q}}(\gamma)$ channel. Conclusions are given in Section 4 .

\section{The $\mathrm{e}^{+} \mathrm{e}^{-} \rightarrow \mu^{+} \mu^{-}(\gamma)$ radiative return events}

\subsection{The determination of $\sqrt{s^{\prime}}$}

For a centre-of-mass energy, $\sqrt{s}$, above the $\mathrm{Z}$ resonance the differential distribution of the invariant mass of the $\mu^{+} \mu^{-}$pair, $\sqrt{s^{\prime}}$, has two distinct peaks. One is a peak at $\sqrt{s^{\prime}} \approx \sqrt{s}$ and the other is at $\sqrt{s^{\prime}} \approx \mathrm{M}_{\mathrm{Z}}$. The latter peak is formed by the radiation of one or more photons from the incoming electron or positron, reducing the effective invariant mass of the $\mathrm{e}^{+} \mathrm{e}^{-}$system to that of an on-shell Z, where the cross-section for s-channel $\mathrm{Z}$ exchange is large.

The lineshape at the $\mathrm{Z}$ has been studied in great detail at LEP1. The measurements of $\mathrm{M}_{\mathrm{Z}}$ and $\Gamma_{\mathrm{Z}}$ have been made to $\mathrm{MeV}$ precision [13]. Using the knowledge of the resonance, combined with that of QED initial-state radiation, one can hope to model the radiativereturn peak to similar precision [14].

If a variable $x=\sqrt{s^{\prime} / s}$ is defined, a distribution of $x$ will have some value, $x_{\mathrm{Z}}$, at the position of the radiative-return peak. In a distribution of $\sqrt{s^{\prime}}$, the position of the radiative return peak is always at some value $\left(\sqrt{s_{\mathrm{Z}}^{\prime}} \simeq \mathrm{M}_{\mathrm{Z}}\right)$, independent of the initial centre-of-mass energy. Therefore, if $\sqrt{s_{Z}^{\prime}}$ is known, a measurement of $x_{\mathrm{Z}}$ can be used to calculate $\sqrt{s}$ from the expression:

$$
\sqrt{s}=\frac{\sqrt{s_{\mathrm{Z}}^{\prime}}}{x_{\mathrm{Z}}}
$$

However, the measurement of $x_{\mathrm{Z}}$ must be independent of $\sqrt{s}$ for this to be a valid method of evaluating $\sqrt{s}$. This is true for radiative $\mu^{+} \mu^{-}$events, which are consistent with only one hard ISR photon being radiated collinear to the beams.

For this simple case of planar events $s^{\prime}$ can be expressed in terms of only $s$ and the energy of the radiated photon, $E_{\gamma}$ :

$$
s^{\prime}=s-2 E_{\gamma} \sqrt{s} .
$$

Using 4-momentum conservation, $E_{\gamma}$ can be written in terms of the polar angles (defined relative to the incoming electron beam) of the muons and $\sqrt{s}$ alone:

$$
E_{\gamma}=\frac{\left|\sin \left(\theta_{\mu^{+}}+\theta_{\mu^{-}}\right)\right| \sqrt{s}}{\sin \left(\theta_{\mu^{+}}\right)+\sin \left(\theta_{\mu^{-}}\right)+\left|\sin \left(\theta_{\mu^{+}}+\theta_{\mu^{-}}\right)\right|},
$$

where $\theta_{\mu^{-}}$and $\theta_{\mu^{+}}$are the polar angles of the momentum vectors of the muon and antimuon, respectively. Substituting for $E_{\gamma}$ in equation (1), leads to an expression for $x$ :

$$
\sqrt{\frac{s^{\prime}}{s}}=x=\sqrt{\frac{\sin \left(\theta_{\mu^{+}}\right)+\sin \left(\theta_{\mu^{-}}\right)-\left|\sin \left(\theta_{\mu^{+}}+\theta_{\mu^{-}}\right)\right|}{\sin \left(\theta_{\mu^{+}}\right)+\sin \left(\theta_{\mu^{-}}\right)+\left|\sin \left(\theta_{\mu^{+}}+\theta_{\mu^{-}}\right)\right|}},
$$


in terms of only $\theta_{\mu^{+}}$and $\theta_{\mu^{-}}$, as desired.

To investigate the possibility of a systematic discrepancy between $\sqrt{s}$ from the method and that from the standard LEP measurement, it is also useful to be able to combine data from many years. A quantity is defined that expresses the difference between the centre-of-mass energy by the method of using radiative-return events and the value from LEP,

$$
\Delta \mathrm{E}_{\mathrm{CM}}=\sqrt{s}_{\text {ANGLES }}-\sqrt{s}_{\mathrm{LEP}}=\frac{\mathrm{M}_{\mathrm{Z}}}{x}-\sqrt{s}_{\mathrm{LEP}}
$$

If the two methods agree, this should give a distribution, centred around zero, that takes the form of the radiative-return peak. A fit can be performed to this distribution and a central value, giving the overall difference, extracted.

\subsection{Event selection}

Initially the standard selection for dimuon events, as used in the cross-section and asymmetry measurements, was applied. A detailed description of the selection can be found in [15]. To reject dimuon events which are not consistent with a single ISR photon down the beam pipe, further cuts were made:

- it was required that no photons were seen within the electromagnetic calorimetry. For this study the calorimetry includes the low-angle luminosity detectors, STIC, as well as the barrel and forward regions, collectively called ECAL. Photons of energy greater than $500 \mathrm{MeV}$ in the STIC, and greater than $2 \mathrm{GeV}$ in the ECAL were considered. This cut removed events with a high transverse-energy photon;

- the acoplanarity ${ }^{1}$ of the two muons with respect to the beam axis was required to be less than 0.1 rad.;

- the ratio of the absolute value of the missing longitudinal momentum (i.e. net momentum of the two muons along the electron beam direction) to the missing energy (i.e. the nominal LEP2 centre-of-mass energy minus the sum of the two muon energies) in the event had to be greater than 0.8 . This selection cut is effective against multiple ISR photons.

The latter two selections also reduce backgrounds from $\tau^{+} \tau^{-}$, four-fermion events and from cosmic rays. This leaves a background level of approximately $2 \%$ in the data set used in this study.

\subsection{Fitting the radiative return peak}

The selected events were fitted to the expected shape of the differential cross-section in $\Delta \mathrm{E}_{\mathrm{CM}}$ using an unbinned maximum log-likelihood fit. The function, $f(x)$, used is:

$$
f(x)=\frac{\mathcal{N} x^{4}}{\left(x^{2}-x_{\mathrm{Z}}^{2}\right)^{2}+\left(\Gamma_{\mathrm{Z}}^{\mathrm{FIT}} x / \mathrm{M}_{\mathrm{Z}}\right)^{2}} \cdot\left(1+\mathcal{B}_{1} / x+\mathcal{B}_{2} / x^{2}\right),
$$

where $\mathcal{N}, x_{\mathrm{Z}}, \Gamma_{\mathrm{Z}}{ }^{\mathrm{FIT}}, \mathcal{B}_{1}$ and $\mathcal{B}_{2}$ are parameters determined by fitting. This is an empirical function which fits well the shape of the simulated data. The package MINUIT [16] was used to maximise the likelihood function.

Events generated by KK [8] and passed through the DELPHI detector simulation package were used to calibrate the fit. It was found that the central fitted value, calculated

${ }^{1}$ The acoplanarity is defined as the complement of the angle, in the plane transverse to the beam, between the two tracks. 
from the parameter $x_{\mathrm{Z}}$, did not correspond to 0 , but was systematically shifted to lower values of $\Delta \mathrm{E}_{\mathrm{CM}}$, i.e. lower values of $\sqrt{s}$. A number of different samples at several centreof-mass energies were used to evaluate the size of this bias. The bias was found to be independent of energy over the range of energies considered. Thus the bias from the combination of all the energies is used as the correction to the final fitted central value from data.

The function (5) was fitted to DELSIM samples for KK events generated at centreof-mass energies at or close to those of the data. The result of the fit to all simulated data is shown in Figure 1. The bias from this fit was found to be $\Delta \mathrm{E}_{\mathrm{CM}}=-0.057 \pm$ 0.022 (stat.) $\mathrm{GeV}\left(\chi^{2} /\right.$ N.D.o.F. $\left.=26.8 / 25\right)$.

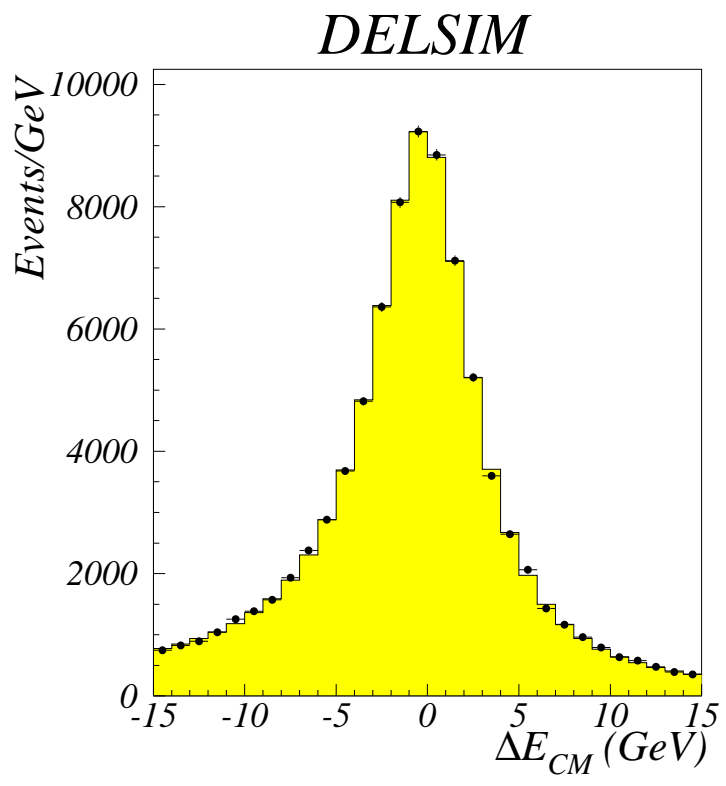

Figure 1: A fit to the $\Delta \mathrm{E}_{\mathrm{CM}}$ distribution (see Section 2.1) of fully-simulated $\mathrm{e}^{+} \mathrm{e}^{-} \rightarrow \mu^{+} \mu^{-}(\gamma)$ events using the KK generator [8], corresponding to an effective luminosity of about $86 \mathrm{fb}^{-1}$. The points show the simulated data, and the shaded histogram is the fitted function.

\subsection{Results}

From the 1997-2000 data sets 600 events were selected and the $\Delta \mathrm{E}_{\mathrm{CM}}$ distribution was fitted with equation (5), with the parameters $\mathcal{B}_{1}$ and $\mathcal{B}_{2}$ fixed at their values obtained from simulation. The fit results at each energy point are shown in Figure 2. Since, within the large statistical errors, there is no apparent dependence on energy, the 1997-2000 data sets were combined. The fit is shown in Figure 3. The result of the fit was:

$$
\Delta \mathrm{E}_{\mathrm{CM}}=+0.184 \pm 0.150 \text { (stat.) } \mathrm{GeV}\left(\chi^{2} / \text { N.D.o.F. }=8.6 / 12\right) .
$$

The statistical error was verified using the "bootstrap" method [17]. In this method, random subsamples of simulated events of the same size as the real sample size are selected and for each of them the result is computed in the same way as for the real sample. This gives some distribution of the resulting value. The spread of this distribution was compatible with the statistical error assigned by MINUIT (i.e. the "pull value" was found 


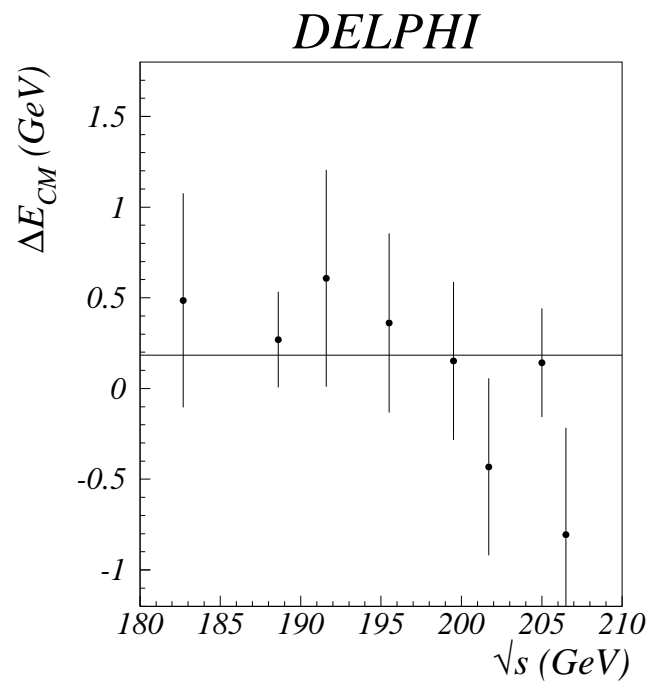

Figure 2: Measured centre-of-mass energy shift at each energy point from $\mathrm{e}^{+} \mathrm{e}^{-} \rightarrow \mu^{+} \mu^{-}(\gamma)$ events. The error bars show the size of the statistical error. The line represents the fit to all energies combined.

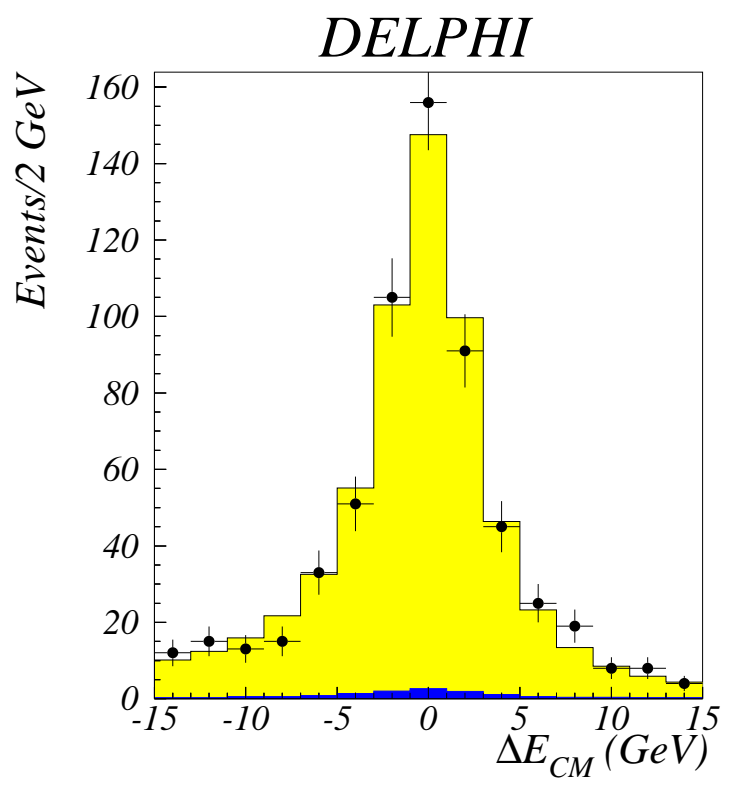

Figure 3: $\Delta \mathrm{E}_{\mathrm{CM}}$ value for data (points) corresponding to the combined sample of $\mathrm{e}^{+} \mathrm{e}^{-} \rightarrow \mu^{+} \mu^{-}(\gamma)$ events collected from 1997-2000. The fitted function is shown by the light grey histogram. The dark grey histogram shows the expected background. The integrated luminosity is $594 \mathrm{pb}^{-1}$.

to be 1.01). However the average statistical uncertainty on the centre-of-mass energy of the samples was found to be $0.172 \mathrm{GeV}$, somewhat larger than that found with the real sample. 


\subsection{Systematics}

The systematic uncertainties are presented below. They include contributions from the modelling of the ISR spectrum as well as effects expected from reconstruction and from the LEP beam parameters.

\subsubsection{Systematics from the bias}

The bias estimation has a systematic uncertainty on $\Delta \mathrm{E}_{\mathrm{CM}}$ of $\pm 22 \mathrm{MeV}$, due to the limited statistics of fully-simulated events.

\subsubsection{Systematics from radiative photonic correction modelling}

The effects of the QED modelling were studied at generator level using the KK [8] program. The KK generator calculates photon emission from initial beams and outgoing fermions to second order, and includes ISR $\otimes$ FSR interference also to this order.

To make some estimate of the magnitude of the effect of imperfections in the radiative photon modelling on the bias, samples of 500,000 KK events were generated at each of the centre-of mass energies used and fitted with the function given in equation (5). Following the advice given by the authors of KK [8], an estimate of the uncertainty of the ISR modelling was obtained by weighting the events generated with the default CEEX2 $\left(\mathcal{O}\left(\alpha^{2}\right)\right)$ approximation to correspond to the CEEX1 $(\mathcal{O}(\alpha))$ approximation, and fitting equation (5) to the weighted events. Half the difference in the resulting bias was taken as the systematic uncertainty due to this effect. It amounted to $2.5 \pm 0.7 \mathrm{MeV}$ on $\Delta \mathrm{E}_{\mathrm{CM}}$.

\subsubsection{Mismeasurement of the DELPHI aspect ratio}

The aspect ratio is defined as the ratio of the length to the width of the detector. It is limited to the precision to which the position and dimensions of the Vertex Detector (VD) can be measured. The effect of a mismeasurement of the aspect ratio is to introduce a bias on the measurement of the polar angle, $\theta$. A positive (negative) bias on $\tan \theta$ would systematically increase (decrease) the opening angle of the two muons and lead to an underestimate (overestimate) of $\sqrt{s}$.

The correspondence of hits in overlapping silicon modules is sensitive to a misalignment of the Vertex Detector. In fact the study of these overlaps constitutes an essential part of the procedure for the alignment of the Vertex Detector. The alignment was carried out independently for each year of operation. A careful analysis of the data of all years shows that a residual bias of $6 \times 10^{-5}$ can be excluded at the $95 \%$ confidence level. However a study of the behaviour of the DELPHI silicon modules [18] shows that the centre of charge of the holes (and electrons) differs from the mid-plane of the detectors by $10-20 \mu \mathrm{m}$. In the alignment procedure this difference would be automatically accounted for. However much less is known about the displacement of the electrons (used in the measurement of $z$, along the beam axis, and hence $\theta$ ) since the necessary overlaps are much fewer. Taking $\pm 10 \mu \mathrm{m}$ as an effective uncertainty on the location of the sensitive region of the detectors, and allowing for the fact that the polar angles of some tracks are determined from signals in the silicon detectors themselves (without using the interaction point as a constraint) it is concluded that a reasonable estimate of the aspect ratio uncertainty is $3 \times 10^{-4}$. Such a bias would correspond to a shift of $\Delta \mathrm{E}_{\mathrm{CM}}$ of about $\pm 29 \mathrm{MeV}$. 


\subsubsection{Systematics from LEP beam parameters}

In general the colliding $\mathrm{e}^{+}$and $\mathrm{e}^{-}$are not collinear at the interaction point, and their energies are not equal. This leads to a boost of the centre-of-mass system relative to DELPHI and in an individual event will lead to a change in the fitted centre-of-mass energy. However, averaged over all event orientations, the effects are much reduced. Similarly, inequality of the beam energies or a tilt of the beams with respect to the DELPHI coordinate system will lead to errors in the fitted energies. Studies at generator level with beam acollinearities and tilts up to $500 \mu \mathrm{rad}$ and beam energy differences up to $100 \mathrm{MeV}$, have shown that the overall effects would be approximately $1 \mathrm{MeV}$. However the spread in energies of the electron and positron beams, which can give an rms spread in the centre-of-mass energy of $\pm 250 \mathrm{MeV}$ at high energy, results in a correction of $6 \mathrm{MeV}$.

\subsubsection{Muon polar angle and momentum reconstruction}

Studies at generator level showed that random errors in the muon polar angle due to reconstruction inaccuracies in DELPHI lead to systematic shifts of the fitted energy. In principle, if correctly estimated, these are taken into account in the simulation. The selection on energy and momentum imbalance also makes use of the measured momenta of the muons, so in principle is affected by bias or additional smearing in this quantity. However it was estimated that the overall effect of these imperfections led to an uncertainty in the centre-of-mass energy of less than $2 \mathrm{MeV}$.

\subsubsection{Backgrounds}

Backgrounds from four-fermion and $\tau$-pair production have been included in the simulated samples from which the bias was calculated. Inclusion of the $2 \%$ estimated backgrounds changed the bias by $0.4 \mathrm{MeV}$ on $\Delta \mathrm{E}_{\mathrm{CM}}$, with a statistical uncertainty of $\pm 4 \mathrm{MeV}$, due to the size of the samples of simulated background events. The latter value was taken as the systematic uncertainty due to the background. The energies of the simulated backgrounds were not varied during the fit. It was estimated that this introduces a negligible additional systematic uncertainty.

\subsubsection{Knowledge of the $\mathrm{Z}$ mass}

As can be seen from equation (4), there will be an uncertainty in the estimate of $\Delta \mathrm{E}_{\mathrm{CM}}$, due to the uncertainty of the $\mathrm{Z}$ mass as measured at LEP1 [13]. The resulting uncertainty from this source amounts to $\pm 4 \mathrm{MeV}$.

\subsubsection{Summary of systematic uncertainties}

Table 1 gives a breakdown of the systematics on $\mathrm{E}_{\mathrm{CM}}$. The total systematic uncertainty on $\Delta \mathrm{E}_{\mathrm{CM}}$ is $38 \mathrm{MeV}$. Thus the dimuon events give a value of:

$$
\Delta \mathrm{E}_{\mathrm{CM}}=+0.241 \pm 0.150 \text { (stat.) } \pm 0.038 \text { (syst.) } \mathrm{GeV} \text {. }
$$




\begin{tabular}{|l||c|}
\hline Source & Error on $\Delta \mathrm{E}_{\mathrm{CM}}(\mathrm{MeV})$ \\
\hline \hline Bias & 22 \\
QED modelling & 3 \\
Aspect ratio & 29 \\
LEP parameters & 6 \\
Angular resolution & 2 \\
Backgrounds & 4 \\
Z mass & 4 \\
\hline \hline Total systematic error & 38 \\
\hline \hline Statistical error & 150 \\
\hline
\end{tabular}

Table 1: The sources and associated values of uncertainties on $\Delta \mathrm{E}_{\mathrm{CM}}$, measured from radiative dimuon events.

\section{The $\mathrm{e}^{+} \mathrm{e}^{-} \rightarrow \mathrm{q} \overline{\mathrm{q}}(\gamma)$ radiative return events}

\subsection{Track and event selection}

Events of the type $\mathrm{e}^{+} \mathrm{e}^{-} \rightarrow \mathrm{q} \overline{\mathrm{q}}(\gamma)$ give rise to a large hadronic multiplicity. The analysis was based mainly on the charged particle tracks reconstructed in each event. Track selection criteria were applied in order to reject tracks originating from secondary interactions as well as poorly measured tracks. The charged particles were required to have momentum larger than $0.4 \mathrm{GeV} / c$, but not exceeding 1.5 times the beam energy, momentum resolution better than $100 \%$, and transverse and longitudinal impact parameters less than $4 \mathrm{~cm}$ and $10 \mathrm{~cm}$, respectively. A more detailed description of the selection can be found in $[15]$.

All hadronic events were required to have at least 7 charged particles and a transverse energy greater than $20 \%$ of the collision energy. The transverse energy was calculated on the basis of the transverse momentum of each selected track and on the electromagnetic showers reconstructed in the HPC and the FEMC with a shower energy above $500 \mathrm{MeV}$. No other cuts were used, in particular for suppression of the WW and ZZ backgrounds, in order to avoid a possible bias to the results. The selection efficiency for hadronic events was slightly less than $80 \%$.

\subsection{Fitting of the radiative return peak}

In the $s^{\prime}$ determination a two-jet configuration was forced. If no ISR photon candidate was observed then the reduced energy was computed from the angles of the jets assuming a photon escaped into the beam pipe. If an isolated energetic photon was observed then the value of $s^{\prime}$ was derived from the combined jet and photon directions, assuming that additional energy could be radiated inside the beam pipe. If no solution was found, the reduced energy was derived from the measured energy of the isolated photon alone.

Figure 4 compares $\sqrt{s^{\prime}}$ distributions between data and simulation [8] around the radiative return peak. In the analysis the interval of $75-105 \mathrm{GeV}$ of the $\sqrt{s^{\prime}}$ distribution was used with bins of width $1 \mathrm{GeV}$. 


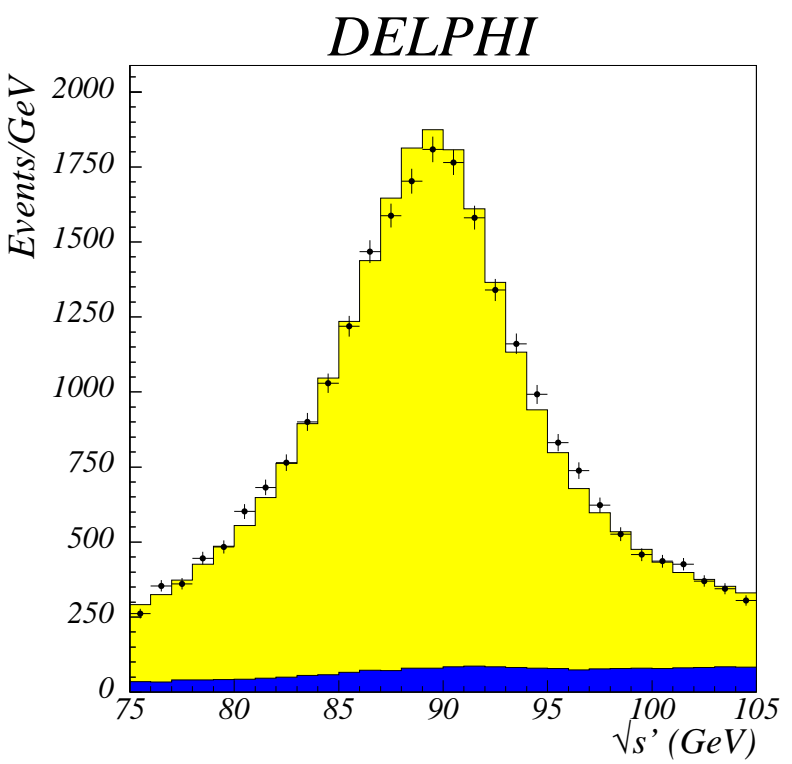

Figure 4: Reduced energy distribution of $\mathrm{e}^{+} \mathrm{e}^{-} \rightarrow \mathrm{q} \overline{\mathrm{q}}(\gamma)$ events. Points represent the data, light grey area the signal simulation, dark grey area the background simulation. The integrated luminosity is approximately $600 \mathrm{pb}^{-1}$. The distributions combine all energy points $183-207 \mathrm{GeV}$. 


\subsubsection{Method of Monte Carlo reweighting}

The method of this analysis was to compare the Z-boson mass, $\mathrm{M}_{\mathrm{Z}}$, measured from data, to its nominal value and to find the centre-of-mass energy shift, $\Delta \mathrm{E}_{\mathrm{CM}}$, from their difference, $\Delta \mathrm{M}_{\mathrm{Z}}$, using:

$$
\Delta \mathrm{E}_{\mathrm{CM}}=-\frac{\Delta \mathrm{M}_{\mathrm{Z}}}{\mathrm{M}_{\mathrm{Z}}} \cdot \mathrm{E}_{\mathrm{CM}} .
$$

The approach used for the $\mathrm{M}_{\mathrm{Z}}$ determination is based on varying the $\mathrm{M}_{\mathrm{Z}}$ value in simulation and finding the value which provides the best agreement of the $\sqrt{s^{\prime}}$ distribution between data and simulation. $\mathrm{M}_{\mathrm{Z}}$ was varied by reweighting the simulation using a BreitWigner-like function $f\left(s^{\prime}, \mathrm{M}_{\mathrm{Z}}\right)$ :

$$
\begin{gathered}
\text { weight }\left(s^{\prime}, \mathrm{M}_{\mathrm{Z}}^{\text {new }}\right)=\frac{f\left(s^{\prime}, \mathrm{M}_{\mathrm{Z}}^{\text {new }}\right)}{f\left(s^{\prime}, \mathrm{M}_{\mathrm{Z}}^{\text {nom }}\right)}, \\
f\left(s^{\prime}, \mathrm{M}_{\mathrm{Z}}\right)=\frac{\left(1+P \cdot\left(\sqrt{s^{\prime}}-\mathrm{M}_{\mathrm{Z}}\right)\right) \cdot s^{\prime}}{\left(s^{\prime}-\mathrm{M}_{\mathrm{Z}}^{2}\right)^{2}+\left(s^{\prime} \cdot \Gamma_{\mathrm{Z}} / \mathrm{M}_{\mathrm{Z}}\right)^{2}},
\end{gathered}
$$

where $P$ is a skewness parameter (set to $0.01 \mathrm{GeV}^{-1}$ ), $\sqrt{s^{\prime}}$ is the generated reduced energy in the given event, $\mathrm{M}_{\mathrm{Z}}{ }^{\text {nom }}$ the nominal value of $\mathrm{M}_{\mathrm{Z}}$ and $\mathrm{M}_{\mathrm{Z}}{ }^{\text {new }}$ the new value of $\mathrm{M}_{\mathrm{Z}}$. This particular form of the function $f\left(s^{\prime}, \mathrm{M}_{\mathrm{Z}}\right)$ was chosen because it describes well the shape of the $\mathrm{Z}$ mass peak of the generated $\sqrt{s^{\prime}}$ distribution, and this is used to set the value of $P$.

\subsubsection{Fitting simulation to data}

The determination of $\mathrm{M}_{\mathrm{Z}}$ was performed by fitting simulation to data using a loglikelihood method. The log-likelihood of the real distribution, given the simulated $\sqrt{s^{\prime}}$ distribution, was computed as:

$$
\mathcal{L}=2 \sum_{i=1}^{n}\left(f_{i}-y_{i} \ln f_{i}\right)
$$

where $n$ is the number of bins in the distribution histogram, $y_{i}$ and $f_{i}$ are the content of the $i$-th bin in the real and simulated distributions, respectively.

For a cross-check a chi-square method was used as well. The chi-square of the difference was computed as:

$$
\chi^{2}=\sum_{i=1}^{n}\left(\frac{f_{i}-y_{i}}{\sqrt{y_{i}}}\right)^{2} .
$$

When the simulation has been reweighted to a new $\mathrm{M}_{\mathrm{Z}}$ value the content of the simulated distribution $f_{1} \ldots f_{n}$ was recomputed accordingly. By a detailed scan, the dependence of $\chi^{2}$ and $\mathcal{L}$ on $\mathrm{M}_{\mathrm{Z}}$ was found to be quadratic over the region of interest. It can be written as:

$$
\chi^{2}\left(\mathrm{M}_{\mathrm{Z}}\right)=\chi_{\min }^{2}+\frac{\left(\mathrm{M}_{\mathrm{Z}}-\mathrm{M}_{\mathrm{Z}}^{\min }\right)^{2}}{\sigma^{2}} .
$$

Three points are needed to find the minimum of the parabola (8). The most convenient choice of points is $\mathrm{M}_{\mathrm{Z}}-0.5 \mathrm{GeV}, \mathrm{M}_{\mathrm{Z}}$ and $\mathrm{M}_{\mathrm{Z}}+0.5 \mathrm{GeV}$. After reweighting the simulation to $\mathrm{M}_{\mathrm{z}}-0.5 \mathrm{GeV}$ and $\mathrm{M}_{\mathrm{z}}+0.5 \mathrm{GeV}$ and computing $\mathcal{L}$ or $\chi^{2}$ at all three points it is straightforward to compute $\mathrm{M}_{\mathrm{Z}}{ }^{\min }$ as well as $\sigma$, which gives the statistical error on $\mathrm{M}_{\mathrm{Z}}{ }^{\text {min }}$. 


\subsection{Corrections in the forward region}

One of the main problems to be dealt with in the analysis was a noticeable excess of tracks at low polar angles (forward tracks) in data as compared to simulation. The effect is shown in Figure 5a. The most likely cause of this effect is an underestimation in the simulation of the track reconstruction efficiency for low-momentum particles at low polar angles.

A tuning was applied to the data in order to correct this discrepancy. It consisted in removing some fraction of forward tracks on a random basis. A track could be removed from an event with some probability $\mathcal{P}$ computed from a ratio between the number of simulated $N_{\text {sim }}$ and real $N_{\text {real }}$ tracks:

$$
\mathcal{P}=1-\frac{N_{\text {sim }}}{N_{\text {real }}}
$$

In computing the correction it was taken into account that the size of the discrepancy depends on the track momentum and polar angle. The momentum range, as well as polar angle range, were split into a certain number of bins. The ratio $N_{\text {sim }} / N_{\text {real }}$ was computed in each bin and the correction was applied accordingly.

For computation of the correction ratios $N_{\text {sim }} / N_{\text {real }}$, the data collected in calibration runs at the $\mathrm{Z}$ peak were used. Such data are available for every year of LEP2 data taking. They provide much more statistical power for computation of the tuning coefficients than the high energy data, and the corrections are uncorrelated with the LEP2 beam energy.

Figure $5 \mathrm{~b}$ shows the polar angle distribution after the correction is applied. Some residual difference still remains because the size of the discrepancy in high energy and in on-peak data was slightly different.

This correction changed significantly the results because radiative events are boosted forward and therefore are sensitive to changes in forward tracking. The measured average shift in the centre-of-mass energy changed as a result of the correction from $+206 \mathrm{MeV}$ to $-116 \mathrm{MeV}$.

It should be noted that this effect does not influence the muon-pair result. The defect in the simulation would show itself as a difference in efficiency between simulation and data. However as part of the dimuon analysis, the detection efficency in simulation is tuned to that measured in data. Furthermore the effect is concentrated at low track momenta. Hence the results presented in Section 2 are not affected.

\subsection{Results}

The results obtained from fitting simulation to data by the log-likelihood method at each energy point are shown in Figure 6. The average shift of the measured centre-of-mass energy with respect to the LEP value is found to be $-116 \pm 106$ (stat.) MeV.

In the analysis the statistical error on $\mathrm{M}_{\mathrm{Z}}{ }^{\min }$ is computed as $\sigma$ from equation (8). In order to verify that it gives a correct estimate of the statistical error, two other methods of the statistical error determination were used.

The first one is the so-called "Jackknife" method [19]. It describes the measurement of the change in the result if one single event is taken out of the sample. If one event is removed out of the bin $i$, the change of the result $R$ is $\Delta_{i}=R-R_{i}$. The variance $\sigma_{R}{ }^{2}$ of the result is then equal to the sum of $\Delta_{i}{ }^{2}$ over all bins times the number of entries $y_{i}$, $\sigma_{R}^{2}=\sum_{i} \Delta_{i}^{2} \cdot y_{i}$. Thus $\sigma_{R}$ should give the same error on $\mathrm{M}_{\mathrm{Z}}$ as before.

The statistical error was also checked using the "bootstrap" method [17] described in Section 2.4. 

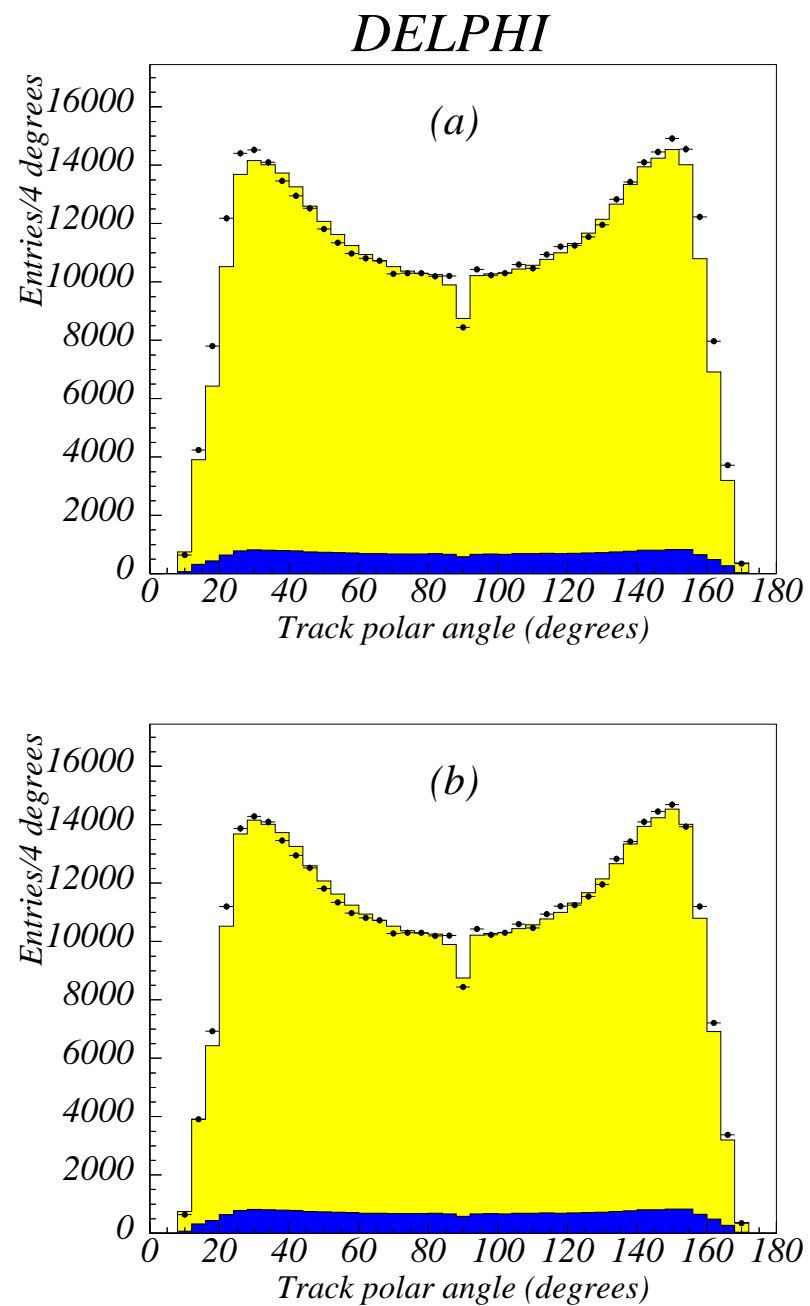

Figure 5: Distribution of track polar angle of radiative return $\mathrm{e}^{+} \mathrm{e}^{-} \rightarrow \mathrm{q} \overline{\mathrm{q}}(\gamma)$ events (a) before and (b) after corrections in the forward region. Points represent data, light grey area the signal simulation, dark grey area the background simulation. The distributions combine all energy points $183-207 \mathrm{GeV}$.

Statistical errors computed with the methods described above were found to be consistent within a few percent with the error computed from equation (8).

The contribution of the statistics of the Monte Carlo sample amounts to an uncertainty of $\pm 14 \mathrm{MeV}$ on $\Delta \mathrm{E}_{\mathrm{CM}}$.

\subsection{Systematics}

\subsubsection{Uncertainty on corrections in the forward region}

The stability of the $\mathrm{E}_{\mathrm{CM}}$ determination was studied using alternative methods for the determination of $\sqrt{s^{\prime}}$ (using also neutrals or a kinematic fit). A correction procedure based on the high energy data rather than calibration data at the $\mathrm{Z}$ peak was also tried, although this could not be used for a result since the correction is not independent of the assumed centre-of-mass energy. A reweighting of tracks in data, rather than their removal, was also used. Considering the spread of the results, and also the size of remaining data- 


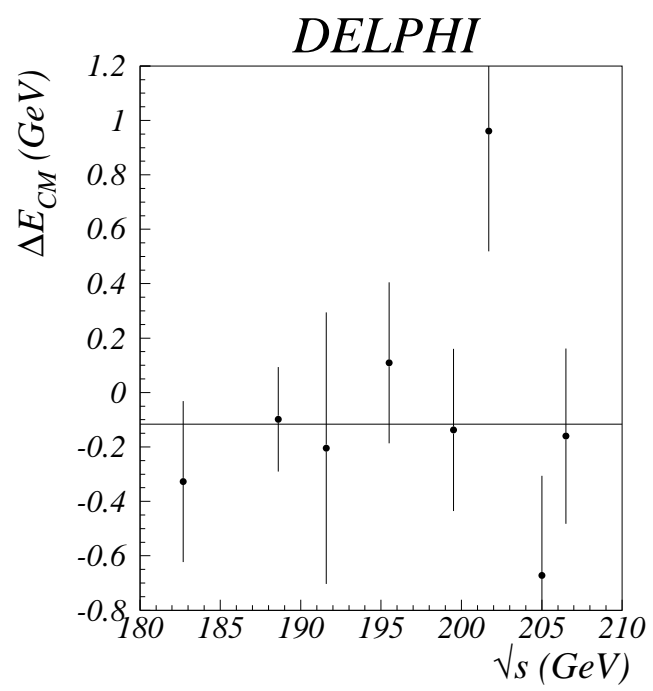

Figure 6: Measured centre-of-mass energy shift at each energy point from $\mathrm{e}^{+} \mathrm{e}^{-} \rightarrow \mathrm{q} \overline{\mathrm{q}}(\gamma)$ events. The error bars show the size of the statistical error. The line represents the average $\left(\chi^{2} /\right.$ N.D.o.F. $\left.=9.4 / 7\right)$.

simulation discrepancies (for example those visible in Figure 5b) it was estimated that a systematic uncertainty of $\pm 120 \mathrm{MeV}$ on $\mathrm{E}_{\mathrm{CM}}$ should be assigned, due to the forward track correction and other imperfections in the simulation.

\subsubsection{ISR modelling uncertainty}

To study the uncertainty related to ISR modelling in simulation, two $\mathrm{e}^{+} \mathrm{e}^{-} \rightarrow \mathrm{q} \overline{\mathrm{q}}(\gamma)$ Monte Carlo samples, made with different generators, were compared. One sample was generated with KK and another one with PYTHIA 6.153.

The comparison revealed no statistically significant effect. When one simulation was fitted with the other, the deviation of $\mathrm{M}_{\mathrm{Z}}$ from the nominal value was found to be consistent with zero. The statistical error of this measurement was approximately $20 \mathrm{MeV}$ on $\mathrm{E}_{\mathrm{CM}}$.

In addition, as for the dimuon events, studies were made at generator level using the KK generator. Samples of 100,000 $\mathrm{e}^{+} \mathrm{e}^{-} \rightarrow \mathrm{q} \overline{\mathrm{q}}(\gamma)$ events were generated at each of the energies used. Half of the difference between the CEEX2 and CEEX1 approximations amounted to $0.2 \pm 0.4 \mathrm{MeV}$ on $\Delta \mathrm{E}_{\mathrm{CM}}$. However in the DELPHI simulation, events are generated with $\mathrm{KK}$ without ISR $\otimes \mathrm{FSR}$ interference. The hadronisation and FSR were generated using PYTHIA. The effect of removing ISR $\otimes$ FSR in KK-generated events amounted to a change in $\Delta \mathrm{E}_{\mathrm{CM}}$ of $4.4 \pm 5.4 \mathrm{MeV}$.

The uncertainty connected with the ISR modelling was therefore taken as $\pm 6 \mathrm{MeV}$ on $\Delta \mathrm{E}_{\mathrm{CM}}$.

\subsubsection{Fragmentation uncertainty}

A similar approach was used for estimating the fragmentation uncertainty. A sample generated with KK and hadronised with PYTHIA was compared to a sample generated with KK and hadronised with ARIADNE [12]. Both PYTHIA and ARIADNE had been consistently tuned to the DELPHI data. Fitting one simulation to the other gave a few 
$\mathrm{MeV}$ shift in $\mathrm{M}_{\mathrm{Z}}$, with the statistical error on the difference of about $10 \mathrm{MeV}$. Therefore the fragmentation uncertainty was estimated to be $\pm 20 \mathrm{MeV}$ on $\mathrm{E}_{\mathrm{CM}}$.

\subsubsection{Four-fermion background uncertainty}

The majority of the four-fermion background is concentrated at high values of $s^{\prime}$. Around the $\mathrm{Z}$ mass peak the background amounts to 8-10\% (depending on energy point) of the signal and it has an almost flat distribution with $s^{\prime}$ (see Figure 4). Thus, a bias in the background will affect the results only weakly.

The uncertainty due to four-fermion background was estimated by scaling the background cross-sections by $\pm 5 \%$. It changed the result by $\pm 12 \mathrm{MeV}$. It was concluded that a $10 \mathrm{MeV}$ error is sufficient to cover the background uncertainty. The energies of the simulated backgrounds were not varied during the fit. It was estimated that this introduces a negligible additional systematic uncertainty.

\subsubsection{Aspect ratio uncertainty}

As discussed in Section 2.5.3, uncertainties in the aspect ratio can bias the reconstruction of jet angles and therefore the $s^{\prime}$ determination as well.

Its effect on the centre-of-mass energy measurement was checked by introducing in simulation an artificial aspect ratio bias of a size of $3 \times 10^{-4}$. It consisted in scaling the longitudinal component of the momenta of charged particle tracks by this amount. The resulting change of the measured centre-of-mass energy was found to be $24 \mathrm{MeV}$. This value was taken as the aspect ratio uncertainty.

\subsubsection{Systematics from LEP beam parameters}

The effects of the LEP beam parameters on the energy measurement were taken to be the same as those found in the generator studies of dimuon events, namely an uncertainty of $\pm 6 \mathrm{MeV}$ on $\Delta \mathrm{E}_{\mathrm{CM}}$.

\subsubsection{Knowledge of the $\mathrm{Z}$ mass}

As in the dimuon analysis, there will be an uncertainty in the estimate of $\Delta \mathrm{E}_{\mathrm{CM}}$ of $\pm 4 \mathrm{MeV}$, due to the uncertainty of the $\mathrm{Z}$ mass as measured [13] at LEP1.

\subsubsection{Error summary}

Table 2 summarizes all contributions to the error of the measurement. The total systematic uncertainty on $\Delta \mathrm{E}_{\mathrm{CM}}$ is $126 \mathrm{MeV}$. Thus the $\mathrm{e}^{+} \mathrm{e}^{-} \rightarrow \mathrm{q} \overline{\mathrm{q}}(\gamma)$ events gave a value of:

$$
\Delta \mathrm{E}_{\mathrm{CM}}=-0.116 \pm 0.106 \text { (stat.) } \pm 0.126 \text { (syst.) GeV }
$$

\section{Conclusions}

High-energy data collected in DeLPHI during the years 1997-2000 were analysed in order to cross-check the LEP energy measurement. The average difference between the centre-of-mass energy measured from radiative return $\mathrm{e}^{+} \mathrm{e}^{-} \rightarrow \mu^{+} \mu^{-}(\gamma)$ events and the energy provided by the LEP Energy Working Group was found to be $+0.241 \pm 0.150$ (stat.) \pm 


\begin{tabular}{|l|c|}
\hline Source & Error on $\Delta \mathrm{E}_{\mathrm{CM}}(\mathrm{MeV})$ \\
\hline \hline Data/MC disagreement & 120 \\
ISR modelling & 6 \\
Fragmentation & 20 \\
Background & 10 \\
Aspect ratio & 24 \\
LEP parameters & 6 \\
MC statistics & 14 \\
Z mass & 4 \\
\hline \hline Total systematic error & 126 \\
\hline \hline Statistical error & 106 \\
\hline
\end{tabular}

Table 2: Contributions to the uncertainty on the centre-of-mass energy shift, as measured from radiative hadronic events.

0.038 (syst.) GeV. The corresponding analysis using the $\mathrm{e}^{+} \mathrm{e}^{-} \rightarrow \mathrm{q} \overline{\mathrm{q}}(\gamma)$ events yielded a difference of $-0.116 \pm 0.106$ (stat.) \pm 0.126 (syst.) GeV. Taking account of correlated systematic errors (aspect ratio, LEP parameters, $\mathrm{Z}$ mass), the two results can be combined to give an overall DELPHI result:

$$
\Delta \mathrm{E}_{\mathrm{CM}}=+0.073 \pm 0.094 \text { (stat.) } \pm 0.065 \text { (syst.) } \mathrm{GeV} .
$$

Thus the DELPHI data are compatible with the values reported by the LEP Energy Working Group [2], who quote values of the LEP centre-of-mass energy with uncertainties ranging from \pm 20 to $\pm 40 \mathrm{MeV}$, depending on the year of operation.

\section{Acknowledgements}

We would like to acknowledge the assistance of the authors of ref. [8] for clarification of several points connected with the KK generator.

We are greatly indebted to our technical collaborators, to former members of the CERN-SL division for their excellent performance of the LEP collider, and to the funding agencies for their support in building and operating the DELPHI detector.

We acknowledge in particular the support of Austrian Federal Ministry of Education, Science and Culture, GZ 616.364/2-III/2a/98, FNRS-FWO, Flanders Institute to encourage scientific and technological research in the industry (IWT), Belgium,

FINEP, CNPq, CAPES, FUJB and FAPERJ, Brazil,

Czech Ministry of Industry and Trade, GA CR 202/99/1362,

Commission of the European Communities (DG XII),

Direction des Sciences de la Matière, CEA, France,

Bundesministerium für Bildung, Wissenschaft, Forschung und Technologie, Germany,

General Secretariat for Research and Technology, Greece,

National Science Foundation (NWO) and Foundation for Research on Matter (FOM), The Netherlands,

Norwegian Research Council,

State Committee for Scientific Research, Poland, SPUB-M/CERN/PO3/DZ296/2000, SPUB-M/CERN/PO3/DZ297/2000, 2P03B 10419 and 2P03B 69 23(2002-2004)

FCT - Fundação para a Ciência e Tecnologia, Portugal, 
Vedecka grantova agentura MS SR, Slovakia, Nr. 95/5195/134, Ministry of Science and Technology of the Republic of Slovenia, CICYT, Spain, AEN99-0950 and AEN99-0761, The Swedish Research Council,

Particle Physics and Astronomy Research Council, UK, Department of Energy, USA, DE-FG02-01ER41155, EEC RTN contract HPRN-CT-00292-2002. 


\section{References}

[1] LeP Energy Working Group, A. Blondel et al.. Eur. Phys. J. C11 (1999) 573.

[2] Lep Energy Working Group, R. Assmann et al., Eur. Phys. J. C39 (2005) 253.

[3] ALEPH Collab., R. Barate et al., Phys. Lett. B464 (1999) 339.

[4] L3 Collab., P. Achard et al., Phys. Lett. B585 (2004) 42.

[5] OPAL Collab., G. Abbiendi et al., Phys. Lett. B. B604 (2004) 31.

[6] DELPHI Collab., P. Aarnio et al., Nucl. Instr. Meth. A303 (1991) 233.

[7] DELPHI Collab., P. Abreu et al., Nucl. Instr. Meth. A378 (1996) 57.

[8] S. Jadach, B.F.L. Ward and Z. Was, Comp. Phys. Comm. 130 (2000) 260.

[9] A. Ballestrero et al., Comp. Phys. Comm. 152 (2003) 175.

[10] E. Accomando and A. Ballestrero, Comp. Phys. Comm. 99 (1997) 270;

E. Accomando, A. Ballestrero and E. Maina, Comp. Phys. Comm. 150 (2003) 166.

[11] T. Sjöstrand et al., Comp. Phys. Comm. 135 (2001) 238.

[12] L. Lönblad, Comp. Phys. Comm. 71 (1992) 15.

[13] The LEP Collaborations ALEPH, DELPHI, L3, OPAL, the LEP Electroweak Working Group and the SLD Heavy Flavour Group, "Precision Electroweak Measurements on the Z Resonance", hep-ex/0509008, submitted to Physics Reports.

[14] Presentation by S. Jadach at the $156^{\text {th }}$ LEP Energy Calibration Meeting held on the $12^{\text {th }}$ June 1998. Minutes can be found at: http://www.cern.ch/LEPECAL/minutes/minutes.html

[15] DELPHI Collab., P. Abreu et al., Eur. Phys. J. C11 (1999) 383.

[16] F. James, "MINUIT Function Minimisation and Error Analysis, Version 94.1", CERN Program Library Long Writeup D506.

[17] B. Efron and R.J. Tibshirani, "An Introduction to the Bootstrap", Chapman and Hall, 1993.

[18] DELPHI Silicon Tracker Group, P. Chochula et al., Nucl. Instr. Meth. A412 (1998) 304.

[19] J. Tukey, Ann. Math. Statist. 29 (1958) 614. 